\title{
Dynamical essence of the basic relations of the special theory of relativity and the origin of fundamental interactions: Phenomenology
}

\author{
Timashev Serge ${ }^{1,2}$ \\ ${ }^{1}$ National Research Nuclear University MEPhI, Moscow, Russia \\ ${ }^{2}$ Karpov Institute of Physical Chemistry, Moscow, Russia
}

\section{Email address:}

serget@mail.ru

\section{To cite this article:}

Timashev Serge. Dynamical Essence of the Basic Relations of the Special Theory of Relativity and the Origin of Fundamental Interactions: Phenomenology. International Journal of Astrophysics and Space Science. Vol. 2, No. 2, 2014, pp. $22-32$.

doi: $10.11648 /$ j.ijass.20140202.12

\begin{abstract}
It is shown that the adoption of the hypothesis that the electromagnetic component of physical vacuum, the vacuum of quantum electrodynamics (QED vacuum), undergoes polarization in the vicinity of elementary particles allows us to comprehend, on the phenomenological level, the physical essence of the basic hypotheses of the special theory of relativity, including the nature of the limitation of the velocity of material objects to the velocity of light in vacuum. The QED vacuum here plays the part of the base medium that governs the development of the fundamental strong and gravitational interactions.
\end{abstract}

Keywords: Phenomenology, Electromagnetic Component of Physical Vacuum, Special Theory of Relativity, Strong and Gravitational Interactions

\section{Introduction}

The special theory of relativity (STR), like any other physical theory, is formulated deductive, i.e., on the basis of postulates. The postulates behind this theory were formulated by Albert Einstein in 1905. The first of them is the principle of relativity according to which all the laws of nature are Lorentz-invariant under transition from one inertial frame of reference to another. The second of the basic postulates is the principle of the constancy of the velocity of light in vacuum, $c$, in all inertial frames of reference, no matter what the velocity of the light source or the observer [1]. An important circumstance here is the indication that vacuum is a physically select base medium in the transmission of electromagnetic signals and translation of material objects from one point in space to another, as well as that the maximum data transfer rate is limited. Naturally what we have in mind here is the electromagnetic component of the physical vacuum - the vacuum of quantum electrodynamics or QED vacuum. Of course, the natural processes of cognition of the Universe can and do apply some corrections and clarifications to the basic postulates of any physical science, specifically the STR. And indeed, the original formulations of the second of the above-mentioned postulates implied that the velocity of light in vacuum was also the ultimate velocity of "propagation of interactions" [1]. However, the recognition of the results of experiments dating from the Einstein-Podolsky-Rosen thought experiment and the analysis of the Bell-Léger inequalities [2-6], which concerned the verification of the basic principles of quantum mechanics, showed that the latter statement required correcting and that the velocity of propagation of interactions could substantially exceed the velocity of light in vacuum. The fixing (measuring with an instrument) the state of one of the two "free" particles being formed that formerly constituted a unified system wherein they existed in an "entangled" or "bound" (as to polarization or spin projection) state [4] unambiguously leads, when measuring the state of the other particle, to the fixing of the alternative state that this particle is required to have according to quantum mechanics, the velocity of such a "remote influence" of the measurement of the state of the former particle on the fixing of the state of the latter one being at least four orders of magnitude in excess of the velocity of light in vacuum [7]. It seems that such an "instant effect" of the first measurement on the result of the second one implies an irreversible, after Weizsäcker [8], alteration, in accordance with the principle of least action, of the entire 
system, including, apart from the two particles and the instrument used to fix the state of the former particle, also the "whole" of vacuum, with its boundary conditions altering during the course of measurement, the neighborhood of the latter, as yet "free", particle being included. And so, one does not have to associate the velocity of light, c, with the ultimate velocity of propagation of interactions.

What is more, astronomical objects in faraway regions of the Universe can move relative to the Earth with velocities exceeding the velocity of light in vacuum [9, 10]. The velocity of an object being observed from the Earth is defined by two components, namely, the velocity of recession resulting from the expansion of the entire space of the Universe and that due to the own peculiar velocity of the object in this expanding space. The dynamics of the general expansion of the Universe is described by the Friedmann equations [11-14], and it is precisely the velocity of this expansion that appears in Hubble's law. The own velocity of an object is its velocity relative to some nearby point at rest in Friedmann's expanding Universe. And as stressed by Silverman [9], it is exactly the value of the sum of the above two recessional velocities that can exceed that of the velocity of light in vacuum, $c$.

It will be demonstrated later in the text that the progress made in astrophysics in the last decade, the ascertainment of the decisive role of the physical vacuum [11-14] in the dynamics of the observable Universe in the first place, gives fresh grounds [15] for the physical comprehension of both the postulates of the STR and the Lorentz transformation based on them [1] and for the establishment of the common essence (genesis) of the fundamental electromagnetic, strong, and gravitational interactions.

\section{QED Vacuum as the Base Medium for the STR}

In the last decade, the notions of physical vacuum have come to be widely used in cosmology. It is exactly the physical vacuum that is associated with "dark energy" in the standard model of the dynamics of the Universe [11-14], based on the Friedmann equations of the general theory of relativity (GTR) that characterize the dynamics of the expanding Universe, global antigravity being inherent in this dark energy which accounts for $73 \%$ of the entire energy of the Universe. Since dark energy is, according to the standard model, evenly "spilled" all over the Universe [11], i.e., distributed uniformly and isotropically on spatial scales from subnuclear to cosmological ones, we will consider the electromagnetic component of this material substance, namely, the QED vacuum, which manifests itself in various physical phenomena [16-19], as the base medium to which our frame of reference will be fixed. This frame of reference is physically select, for it is "related" to Friedmann's space expanding in accordance with the Friedmann equations [11-15] and is characterized by global time common to all points of space and reckoned from the Big Bang [20].

We intend to demonstrate that the introduction of the QED vacuum as such a base medium allows one primarily to discuss issues that relate to the postulates of the STR and are usually aimed at revealing the physical reasons for the limitation of the velocity of material bodies with a rest mass of $\mathrm{m} 0$ to the value of $\mathrm{c}$. Also questioned is the basic Einstein relation for the rest energy $E_{0}$ of such a body: $E_{0}=m_{0} c^{2}$. Indeed, what is the physical reason for the appearance of the velocity of light in vacuum in this relation for a body at rest? The usual answer is that these postulates manifest their adequacy in the agreement between the STR and experimental observations. All the same one usually wants to understand why this is so. However, there are more "inconvenient" questions.

Imagine a thought experiment. Let two identical relativistic particles with a rest mass of $m_{0}$, which are about to collide "head-on", fly to meet each other with the same relativistic velocities of $u$, but do not collide, and encounter instead a 1-cm-thick metal barrier placed in their way between them. The question then arises: What is the relative velocity of these particles prior to their encounter with the barrier; i.e., the velocity at their "approach" to a distance of 1 $\mathrm{cm}$ from each other? And one more question: what is the relative velocity of these particles when there is no barrier between them, so that they collide; i.e., what is their "relative velocity at collision"? The answer to the latter question is well known: the relative velocity of the particles is equal to $c$, which is in complete compliance with the relativistic velocity addition law [1]. It is precisely this result that is demonstrated by numerous accelerator experiments. As for the former question, elementary logic provides the answer: The approach velocity, governed by two independent processes - the approach of the two particles to the metal barrier - is around $2 c$ (naturally the velocity of each particle is somewhat lower than $c$ ).

According to $[15,21]$, the seeming contradiction can be understood as follows. Suppose that each particle, while moving in the physically select base medium - the electromagnetic component of the physical vacuum, polarizes this medium around itself and thus forms a "vacuum polaron". Obviously the polarization of the medium can affect the dynamics of a particle with a mass of $m_{0}$ moving freely in it at a velocity of $u$. It is precisely because the rate of alteration of the physical vacuum surrounding a moving object is limited to the value of $c$ that the movement of material bodies with velocities approaching $c$ in this vacuum proves impossible. Formally this should correspond to an unlimited growth of the "dynamic" mass $E_{u} / \mathrm{c}^{2}$ in the Einstein relation

$$
E_{u}=\eta_{u} m_{0} c^{2}, \eta_{u}=\left(1-\frac{u^{2}}{c^{2}}\right)^{-1 / 2}
$$

where $E_{u}$ is the total energy of a particle having a Lorentz-invariant mass of $m_{0}$ and moving freely with a velocity of $u$. Note that expression (1) can be considered [22] 
as a corollary to the postulated Einstein expression for the rest energy of the particle, $E_{0}=m_{0} c^{2}$, and the relativistic relation for the square of its momentum

$$
\vec{p}^{2}=\frac{1}{c^{2}}\left(E_{u}^{2}-m_{0}^{2} c^{4}\right)
$$

True enough, with such a formalized definition, there remain questions as to the physical reasons for the growth of the kinetic component $E_{u}=\left(\eta_{u}-1\right) m_{0} c^{2}$ of the particle's energy in the relativistic limit as $u \rightarrow c$. It is exactly for these reasons that an alternative opinion exists about the velocity dependence of the mass of relativistic particles [23]. Note also that the concept of "vacuum polaron" introduced above allows one to comprehend the dynamical meaning of the limitation to $c$ of the relative velocity of colliding relativistic particles and thereby answer one of the questions raised above. Indeed, a collision of two "vacuum polarons" implies the formation, at the stage of their interaction, of a unified polarization region common to both particles, the rate of the attendant alteration of the vacuum polarization regions in the vicinity of the colliding particles being limited to the value of $c$. The hypotheses at the root of the special theory of relativity, based on a series of fundamental experiments, thereby become physically meaningful. All what has been said above means a certain "materialization" of the Lorentz transformations, when referring them to the systems manifest in experiments.

The question now arises: What changes occurring in the region of polarization of the QED vacuum in the neighborhood of a material particle moving with a relativistic velocity can be responsible for the appearance of the factor $\eta_{u}$ in expression (1)? It should be noted that this factor was introduced for the first time by Oliver Heaviside in 1889 ([24], p. 35) when making model calculations of the entrainment of the aether, the base medium of the 19th century's science, by a moving charged spherical particle of radius $a$ and mass $m_{0}$. The aether entrainment effect grew stronger in proportion to $\eta_{u}$ as the velocity of the particle increased because of the geometrical displacement of the "Faraday tubes of force" associated with the sphere towards the equatorial plane passing through its center normal to the direction of its motion. In that case, the original spherical geometry of the system - the spherical particle with the Faraday tubes of force issuing normally to its surface, existing at low velocities of the particle, is transformed, if its volume is arbitrarily limited to the radius $R$ equal to a few radii $a$, into an oblate ellipsoid of revolution about the minor axis of an ellipse oriented along the trajectory of the particle, so that

$$
R_{\min }=R \cdot \eta_{u}^{-1}<R_{\max }=R
$$

where $R_{\min }$ and $R_{\max }$ stand for the minor and major half-axes of the oblate ellipsoid of revolution, respectively. Based on this result, J. J. Thomson calculated [24] the momentum of the particle in its surrounding medium and demonstrated that the mass of the particle increased with its velocity in proportion to Heaviside's factor $\eta_{u}$ because of the increase in the total amount of aether entrained by the Faraday tubes of force associated with the moving particle. The subsequent conceptualization of the STR and experimental investigations wholly confirmed the universal role of the factor $\eta_{u}$ in the gamut of relativistic phenomena.

According to the notions being expounded, the QED vacuum - the base medium and physically select frame of reference for all objects of our Universe - is a present-day analog of the aether of the 19th century's science. And therefore, following the general ideas put forward by $\mathrm{O}$. Heaviside and J. J. Thomson, it is but natural to associate the effect of enhancement of the energy of a relativistic particle in the STR with the changes the vacuum polarization region undergoes in the vicinity of this particle in the direction of its motion. One should bear in mind here that the postulated association of the polarization of vacuum in the neighborhood of any material object with the QED vacuum actually implies the openness, in the sense of dynamics, of this object to vacuum. In other words, the properties of any elementary particle are formed upon interaction between its intrinsic essence and the electromagnetic component of the physical vacuum [15].

Naturally such association should be reflected in the conditions at the boundary between the QED vacuum and any material object, including the system "an elementary particle-QED polarization region in its vicinity". When writing down the pertinent boundary conditions - boundary conditions of the 3rd kind $[25,26]$, one should introduce a type I state of the particle boundary proper (an "adsorption" state or "adstate"), whose dynamic variable $\xi(u)$ characterizes the effective relation between the particle and the QED vacuum, and a type II state of the interface characterizing the "activity" of the near-boundary region of the QED vacuum in its interaction with the type I state. Let the dynamic variable $\xi(u)$ characterize the measure (level) of the conditional "lubrication" of the type I state that is necessary for the particle to move in the vacuum medium, whose magnitude decreases (the particle gets partially "stripped") as the velocity $u$ grows higher. According to Weizsäcker's logic [8], the very fact of the transition of the system into a new state is inevitably associated with the dissipativity and irreversibility of this process. But to what extent can actual energy manifest itself in the effects of the QED vacuum for such dissipative and irreversible processes to be initiated? It is well known that effects of this type can be due to a perceptible contribution from the quantum fluctuations of the QED vacuum to the radiation pressure (macroscopic manifestations of such effects were observed by Brooks and co-workers [27]), the static Casimir effect $[17,18]$, as well as the dynamical Casimir effect [28, 29] involving direct transformation of the fluctuations of virtual photons into real photons at the boundaries of objects moving with relativistic velocities. This can offer grounds for the following consideration.

Let us introduce the quantity $k_{1}(u)$ - a rate constant, conditioned by the intrinsic state of the system, for the rate 
of alteration of the polarization region of the QED vacuum in the vicinity of the particle, consequent upon the reduction of the level of "lubrication" (its being "blown-off) as a result of interaction between the particle moving with a velocity of $u$ and the QED vacuum. The alteration of the polarization region of the QED vacuum is also affected by another process characterized by the rate constant $k_{2}$, which consists in the transfer of energy from state II to state I of the particle, the total energy transfer rate increasing with decreasing relative level of "lubrication", $\xi(u)$; i.e., with increasing relative proportion of the "bareness" of the particle, $(1-\xi)$. Each time the polarization region alters with increasing velocity $u$ of the particle, the "resistance" to the motion of the latter grows higher because of the enhancement of its "friction" on virtual photons due to the reduction of the level $\xi(u)$. The QED vacuum acts in point of fact as "reins" on the particle that strives for escape from its polarizing shell, and so as the velocity $u$ of the particle grows higher, so does the potential energy of the system.

Considering what has been said above, the appropriate balance equation for the variable $\xi(u)$ in the stationary case of particle moving in the QED vacuum with a velocity of $u$ may be represented as follows:

$$
\frac{d \xi(u)}{d t}=-k_{1} \xi(u)+k_{2}(1-\xi)=0
$$

so that

$$
\xi(u)=\frac{k_{2}}{k_{1}+k_{2}} .
$$

It is but natural to suppose that the rate constant $k_{1}$ characterizing the loss of energy by "friction" upon interaction with virtual photons must increase with rising velocity $u$. Based on the relations of the STR and the results of pertinent experimental investigations, we assume that $k_{1}=k_{10} \eta_{u}$, where $k_{10} \equiv k_{1}(0)$ and $\eta_{u}$ is Heaviside factor (1). At the same time, the rate constant $k_{2}$ must be independent of $u$. In that case, expression (5) may be written down in the form:

$$
\xi(u)=\frac{k_{2} \eta_{u}^{-1}}{k_{10}+k_{2} \eta_{u}^{-1}}=\frac{k_{2} \sqrt{1-u^{2} / c^{2}}}{k_{10}+k_{2} \sqrt{1-u^{2} / c^{2}}}
$$

so that

$$
\xi(0)=\frac{k_{2}}{k_{10}+k_{2}}, \quad \xi(u) \underset{u \rightarrow c}{\longrightarrow} \frac{k_{20}}{k_{1}} \sqrt{1-u^{2} / c^{2}} .
$$

It is with the reduction of the quantity $\eta_{u}^{-1}$ with increasing velocity of the particle and growing potential energy of the system, consequent upon the disappearance of the "lubrication" needed for the particle to move in the base medium, that is natural to associate the nature of the relativistic growth of the inertial mass and impossibility for an object to move in the medium with the velocity of light, in accordance with Feynman's understanding of relation (1) [23]. This conclusion completely complies with J. J. Thomson's idea that the kinetic perturbations developing in the medium surrounding a moving charged particle are equivalent to the potential (and not kinetic!) energy of the particle and contribute to the growth of precisely this component of the total energy of the system [24].

Within the scope of the ideas being expounded, the motion of a particle with a constant velocity $u$ relative to the base frame of reference fixed to the QED vacuum should naturally be considered not free, but stationary. However, according to the existing tradition going back to E. Mach [30], we will consider natural the above-indicated "assistance" rendered by the QED vacuum to the particle of mass $m$ in maintaining its uniform and rectilinear motion in relation to the base system of reference, without any effect being exerted on it by other bodies or fields, and define motion of this type as inertial. Here we postulate in fact Mach's principle, but in some generalized sense. In the given case, the particle's "inertia" being introduced owes not to all the masses in the Universe [30], but to the action of the electromagnetic component of the physical vacuum on every particle, which conditions the stationary (not "free", irreversible upon time inversion) inertial motion of the particle relative to the base medium - the QED vacuum "related" to Friedmann's space [15]. Such introduction of inertia corresponds to a greater extent with the present-day concepts of the dynamics of the Universe, according to which no more than $4 \%$ of the total energy of the Universe is associated with all the masses existent therein. At that, of course, there remain open questions as to the amount of energy contributed by the QED vacuum to such inertial motion of the particle and the possible dependence of this contribution on the particle's mass and velocity. In connection with the recent discovery of the Higgs boson [31-33], questions can arise about the role the Higgs field plays in the development of inertia in particles. In accordance with the above-said, it can be supposed that the Higgs field imparts to a particle only its mass, while its connection with the QED vacuum, which is responsible for the development of inertia in material objects, is implemented through its interactions that are phenomenologically characterized by the rate constants $k_{1}$ and $k_{2}$.

It is of interest to show how far will remain the notions evolved on the genesis of inertia when considering the motion of a charged particle (for the sake of definiteness, an electron) in a condensed medium at a velocity of $u$ exceeding that of light in this medium; i.e., subject to the condition $u>c / n(\omega)$, where $n(\omega)$ is the refractive index of the medium that depends on the frequency $\omega$ of light. It is well-known that in this case there originates the Vavilov-Cherenkov radiation [34, 35] characterized by a pronounced directivity - the waves of the given frequency $\omega$ are only emitted at a certain angle, $\theta$, to the direction of motion of the system. This angle is defined by the relation $\cos \theta=c / n(\omega) u$. Radiation here is generated by the medium 
outside of an arbitrary channel of radius $r_{u}=\lambda \eta_{u}$ [35], where $\lambda=2 \pi c / \omega$ is the wavelength of light in vacuum, along whose axis moves the particle. It should be noted that the refractive index, as a macroscopic quantity, manifests itself only at distances of $l>\lambda$ from the axis of the channel, which exceeds by several orders of magnitude the characteristic size of the polarization region of the QED vacuum, $a_{B}=\sqrt{2} \hbar / m_{0} c$ (see elsewhere in the text), in the vicinity of the electron causing the generation of the Vavilov-Cherenkov radiation while moving in the medium at a velocity of $u$. This means that the medium exerts no direct effect on the motion of the electron at a superluminal velocity. Two questions arise in this connection. First, can the Vavilov-Cherenkov radiation be associated with the generation of photons as a result of the dynamical Casimir effect $[28,29]$. And secondly, whether or not the energy loss suffered by the QED vacuum in superluminal motion is totally compensated for, and the radiation loss should be attributed to the total dissipative loss by "friction" on the QED vacuum; or the kinetic energy of the particle decreases somewhat when the Vavilov-Cherenkov radiation is generated, as in the case of motion in vacuum [34]. These problems could apparently be resolved in subsequent experiments. To conclude this section, let us dwell upon one more relativistic effect, namely the increase of the lifetime of decaying relativistic particles. We will cite as an example the well-studied decay process $\mu^{-} \rightarrow e^{-}+\widetilde{v}_{e}+v_{\mu}$ in a ring accelerator [36]. The probability of this process is described with a high degree of accuracy by the following relation of the STR:

$$
w=w_{0} \eta_{u}^{-1}=w_{0} \cdot \sqrt{1-\frac{u^{2}}{c^{2}}}
$$

where $w_{0}$ is the decay probability of the particle at rest. It is natural for one to attribute the lowering of the decay probability, because of the parameter $\eta_{u}$ decreasing with increasing velocity $u$ of the particle, to the kinetic difficulties involved in the cardinal alteration of the polarization region of the QED vacuum surrounding the particle prior to its decay. Indeed, such decay becomes possible, provided that quite definite QED vacuum polarization zones are produced in the neighborhood of the electron and electron antineutrino, as well as the muon neutrino, being formed, the particle scattering directions being governed by the laws of conservation of energy and momentum.

While the relativistic mass and lifetime increase effects are real phenomena associated with the polarization dynamics of vacuum in the vicinity of relativistic particles, the Lorentz length contraction effect [1] seems to be purely apparent, associated not with the alterations occurring in the vacuum polarization region in the vicinity of relativistic particles, but with the finiteness of the rate of acquisition of data on successive measurements of the position of an object moving with a relativistic velocity. It is unlikely that the crystal lattice parameters of the solid-phase systems of material objects will alter even in the case of their relativistic motion with respect to the QED vacuum of the Universe.

\section{Physical Essence of the Particle Mass-Energy Relation: Genesis of Nuclear Forces}

Let us impart to our qualitative considerations a model character by associating the polarization of the electromagnetic component of the physical vacuum with the Casimir forces developing in the neighborhood of material objects. A characteristic example of such an association involves the consideration of the Casimir forces in a vacuum gap with a width of $d$ between two ideally smooth metal plates of high electric conductivity $[17,18]$. The Casimir attractive force between such plates, per unit of their surface area, which is due to the amplification of one, the "resonant", frequency of the electromagnetic component of the physical vacuum, given by $c / d$, and suppression of the gamut of the rest of the frequencies of virtual photons, is expressed as [17, $18]$

$$
F_{C}(d)=-\frac{\pi^{2}}{240} \cdot \frac{\hbar c}{d^{4}} .
$$

The validity of expression (7) was experimentally verified while varying the width $d$ of the gap from 50 to $500 \mathrm{~nm}$ [17]. At higher $d$ values the force was difficult to measure with adequate precision.

Let us formally interpolate expression (7) for the Casimir attractive potential from the above-indicated macroscopic gap widths to the sizes of atomic nuclei and elementary particles and consider the Casimir force-associated polarization of the electromagnetic component of the physical vacuum in the neighborhood of a spherical particle of mass $m_{0}$ and radius $a$. The idea of such an interpolation goes back to Casimir (see [37]) who believed that the pertinent forces should stabilize elementary particles, the electron in particular, by retaining its negative charge, thus actually playing the part of the "Poincaré rubber bands" [38] However, calculations of the Casimir forces for a metal sphere showed [39] that the vacuum energy of such a sphere is positive, $U_{a}=0.04618 \hbar c / a$, which means a tendency to expand (repulsive forces are active here). Casimir's idea was therefore abandoned. But one should bear in mind here that in the original Casimir's formulation the polarization of vacuum near a metal surface was only associated with the change in the spectrum of zero-point oscillations due to the tangential component of the electric field vector going to zero on the metal surface. But according to the phenomenological notions being evolved in this work, when a material particle interacts with the electromagnetic component of the physical vacuum, there originates, as stressed above, a "vacuum polaron" as a unified "material object-vacuum" system whose boundary is permeable, opens to vacuum. The representation of an elementary particle or atomic nucleus in the form of a hollow conductive 
sphere in vacuum cannot be considered an adequate model of such a polaron. The Casimir potential of interaction between an elementary particle or atomic nucleus and the electromagnetic component of the physical vacuum, which governs the formation of a "vacuum polaron", must a priori be attractive. Such a result can formally be obtained by solving the Laplace equation for the sphere by the generally accepted method [39], provided that the above-discussed boundary conditions of the 3rd kind $[25,26]$ are introduced, which in the given case provide for the interrelation between the normal component of the electric field vector at the interface and the corresponding derivative of this component with respect to the normal to the surface.

Certain grounds for the hypothesis put forward above can also be seen in the very fact of existence of spin in various nuclei and elementary particles. According to Ohanian [40], the spin of a particle can be treated as a moment developing as a result of circulation of an energy or momentum flux in the field associated with the particle itself. In the case under consideration, this field forms as a self-consistent field upon polarization of the electromagnetic component of the physical vacuum in the region adjoining the particle. It is important to bear in mind [40] that spin here is independent of the internal structure of the particle. In a sense, it is exactly the conditions at the boundary between the physical vacuum and the particle that can be responsible for the particle's spin being a multiple of $\hbar / 2$ or zero in each particular case.

Within the scope of the phenomenological approach being considered, let us select one of the inertial frames associated with the QED vacuum, whose origin coincides with the position of some particle. The expression for the potential energy the particle acquires as a result of amplification within its volume of the QED vacuum frequency equal to $c / a$ and suppression of the gamut of the rest of the frequencies of virtual photons has the form of the "Casimir" attractive potential

$$
U(\vec{r})=-\gamma_{0} \frac{\hbar c}{r}+\frac{\hbar^{2}}{2 m_{0} r^{2}} l(l+1) .
$$

Here $\vec{r}$ denotes the radius-vector (the origin of coordinates coincides with the particle's position), $l$ is the azimuthal quantum number, and $\gamma_{0}$ is a dimensionless parameter characterizing the intensity of the interaction under consideration (the magnitude of this parameter will be determined elsewhere in the text).

Now there arise the natural questions: To what extent is expression (8) justified? How much is the introduction of the idea of a vacuum polarizable on nuclear scales adequate to the hypothesis for the amplification of the select, "resonance", modes and suppression of all the other modes? Can expression (8) be considered a phenomenological interpolation, bearing in mind that corollaries to it can have a definite physical meaning? A basis for getting the electromagnetic component of the physical vacuum and Casimir forces involved in the analysis of phenomena occurring on a nuclear level is offered by the giant dipole resonance phenomenon well known in nuclear physics [41-43]. This phenomenon consists in the excitation of collective electric dipole and more complex modes in nuclei having sufficiently large $(\sim 50 \mathrm{mb})$ gamma absorption cross-sections (experimental data are available for practically all stable isotopes). The very fact of the principal possibility of amplification of some resonance modes of nuclear matter in interaction with electromagnetic field and suppression of a spectrum of "nonresonance" modes points to the possibility of manifestation of the Casimir effect in the atomic nucleus. It is obvious that the effective gamma absorption coefficient in the case of excitation of the $p$-mode of giant dipole resonance, $\alpha_{p \text {-GDR, }}$, must depend on both the fine structure constant of the nucleus, $\alpha_{\mathrm{e}}=q_{\mathrm{e}}{ }^{2} / \hbar c \approx 1 / 137\left(q_{\mathrm{e}}\right.$ is the electron charge), and the strong interaction coupling constant $\alpha_{\mathrm{s}}: \alpha_{p-\mathrm{GDR}}=\alpha_{p-\mathrm{GDR}}\left(\alpha_{\mathrm{e}}, \alpha_{\mathrm{s}}\right)$.

The solution of the Schrödinger equation in a centrally-symmetric field with potential energy (8) has been well known (see, for example, [44], paragraph 36). The energy levels $E(n, l)$ of a discrete spectrum at a potential energy of (8), which reflect the degree of connection between a particle of mass $m_{0}$ and the electromagnetic component of the physical vacuum subject to polarization, are defined as

$$
E\left(n_{r}, l\right)=-\gamma_{0}^{2} \frac{m_{0} c^{2}}{2 n^{2}} \quad n \geq l+1
$$

where $n$ is the principal quantum number. The expression for the "Bohr radius" $a_{\mathrm{B}}$ determining the localization region of the particle in this case has the form

$$
a_{B}=\frac{2 \hbar}{\gamma_{0} m c} .
$$

It follows from expressions (9) and (9a) that at $\gamma_{0}=\sqrt{2}$ the position of the lower level (determined at $l=0$ and $n=1$ ), which characterizes the binding energy between the particle under consideration and the QED vacuum, corresponds in magnitude to the "rest energy" of the particle in the form suggested by Einstein: $E_{0}=m c^{2}$. In that case, $\left.U(\vec{r})\right|_{r=a_{B}}=-m_{0} c^{2}$. It follows from the meaning of expression (9) that it would be more proper to refer to the quantity $E_{0}$ as the "binding energy between the particle and the QED vacuum" than the "rest energy of the particle", the mass defect in nuclear processes simply characterizing the energy released as a result of the difference in energy between the bindings of the original and final products with the vacuum. For this reason, the statements encountered in the literature about the equivalence of mass and energy should be considered incorrect. In what follows, we will treat the localization region of the particle in the physical vacuum, defined by expression (9a), as the characteristic spatial scale of the above-introduced "vacuum polaron". (Traditionally the characteristic spatial scale of a particle is associated with its Compton wavelength $\left.l_{\mathrm{C}}=2 \pi \hbar / m_{0} c\right)$. 
According to expression (9a), the Bohr radius for the proton is $a_{\mathrm{B}}=2.82 \times 10^{-14} \mathrm{~cm}$; i.e., it corresponds to the scale of action of nuclear forces. Therefore, the quantity $\gamma_{0} \hbar c$ that at $\gamma_{0}=\sqrt{2}$ determines, according to expression (8), the potential energy of the attractive interaction between the particle and the QED vacuum, as a result of which the latter gets polarized in the vicinity of the particle, can conditionally be defined as the square of the "strong interaction charge" $q_{\mathrm{s}}$, so that $q_{s}^{2}=\sqrt{2} \hbar c$. It is natural to represent the dimensionless constant $\alpha_{s}$ of such an interaction, by analogy with the fine structure constant, in the form

$$
\alpha_{\mathrm{s}}=q_{\mathrm{s}}^{2} / \hbar c=\sqrt{2} .
$$

In that case, potential energy ( 8$)$ at $l=0$ is determined by the squared strong interaction charge $q_{\mathrm{s}}{ }^{2}$ :

$$
U(\vec{r})=-\sqrt{2} \frac{\hbar c}{r}=-\frac{q_{\mathrm{s}}^{2}}{r}
$$

Such character of interaction with the physical vacuum is typical of any elementary particle. If the particle is structureless (e.g., a lepton) and neutral, expression (9) then exhausts the information significance of potential energy (8a). If such a particle is charged, its Coulomb interactions with other particles found in the same base medium, the physical vacuum, must be examined in the usual way. If the particle has a structure (e.g., a hadron), relation (8a) within the particle can be considered as a "priming" potential energy of nuclear forces characterized by a "nuclear charge" of $q_{\mathrm{s}}$ and a strong interaction coupling constant of $\alpha_{\mathrm{s}}$. Obviously with the nuclear matter "within" such an elementary particle being dynamically mobile, the priming potential of nuclear forces is shielded, and so there develop the effective potentials of "short-range" nuclear forces that decrease exponentially with distance, like the Yukawa potential:

$$
U(\vec{r})=-\frac{q_{s}^{2}}{r} \exp \left(-a_{B s} r\right)
$$

which should be used in the analysis instead of (8a). This agrees with the standard notion of the dynamical nature of the nuclear forces that are usually associated with the exchange of $\pi$-mesons between nucleons.

The unexpectedness of the latter result consists in the physical unity of the electromagnetic and strong interactions that is being actually revealed at the phenomenological level. Since the nature of the Casimir effect is associated with the local spatial changes that the electromagnetic component of the physical vacuum undergoes in the vicinity and inside of a material object, with the resonant amplification of some frequencies characteristic of this object and suppression of the other frequencies contained in the spectrum of the physical vacuum, the nuclear forces in this model constitute the response at nuclear space-time scales of the nuclear matter to the action of the electromagnetic component of the physical vacuum. Obviously this response is extremely peculiar and is governed, on the one hand, by the specific features of the configuration and dynamics of each particular nucleus, and on the other, by its excitation. From this viewpoint one can qualitatively comprehend the above-indicated dependence of the strong interaction coupling constant $\alpha_{\mathrm{s}}$ on the magnitude of excitation [45]. The result obtained, (9), can be considered a "justification" of a sort for the use of expression (8) up to distances corresponding to the size of elementary particles, for it yields physically meaningful results. First of all, it becomes clear that all the objects in our world turn out to be related to the QED vacuum that is being treated as a physically select base medium. Also clear becomes the physical reason for the appearance in the expression for the particle's rest energy $E_{0}$ of a characteristic of this medium, namely, the velocity of light in vacuum, as a parameter entering into definition (8) of the potential, which determines, when multiplied into the Planck constant $\hbar$, the polarization of the particle in the physical vacuum and characterizes the rate of alteration of the conditions of the particle's conjugation with the electromagnetic component of the physical vacuum during its travel in this base medium. As pointed out above, it is exactly the polarization of the particle in the QED vacuum that can be considered a factor of additional stabilization of elementary particles and stable isotopes, capable of keeping the electron, like any other charged elementary particle, from rupture [38].

It is not unlikely that the nature of confinement of quarks within hadrons [45] can also be associated with the Casimir forces. Of interest are also excited states of a particle localized in vacuum. In particular, such levels can be manifest as "resonances", short-lived excited hadron states with characteristic lifetimes in the range $10^{-22}-10^{-24} \mathrm{~s}$ that are formed in $\pi$-meson-nucleon interactions [46].

It is obvious that despite the "connection" being discussed between all material particles and the physical vacuum, the directivity of all local processes involving the so-called "free" particles, i.e., such particles as are related to vacuum only, must be realized in accordance with the principle of least action [47]. This is also true of the processes initiated by the fluctuations of the physical vacuum (emission of a photon by an isolated excited atom [48], radiative decay). And the aforementioned directivity is in fact responsible for the weakening of the connection between the final products of each particular local process and the physical vacuum, the remaining portion of energy being converted into heat (dissipated).

The conclusion drawn about the connection as per expression (9) between material particles and the QED vacuum also complies with the modern-day cosmological notions of the apparent "freezing" of material objects into the expanding space of the Universe [11-14]. It is exactly the fact that every galaxy, every galactic cluster moves as an integral system in the expanding Universe, notwithstanding the relative motions of individual stars, constellations, and 
nebulae as a result of their interactions within each galaxy, that betokens such a "freezing-in". This circumstance stresses once more how natural it is to associate with the expanding Universe the physical vacuum as the base medium and the sole frame of reference with a unified global time common to all objects in the Universe.

It is obvious that given such a base system, the degree of "freezing-in" of cosmological objects increases when they move with relativistic velocities with respect to Friedmann's base frame of reference, so that the polarization region of the vacuum decreases along the direction of their travel and tends to zero as $u \rightarrow c$. And it is precisely with the difficulties of the dynamic alteration of the electromagnetic component of the physical vacuum in the region of its conjugation with material objects moving with relativistic velocities, which lead, according to Feynman's conception of expression (1) [23], to the increase in the inertial mass of cosmologically distant galaxies and galactic clusters moving in the base medium - Friedmann's space, according to [8, 49], that we associate the phenomenon of the so-called "dark matter" which accounts for $23 \%$ of the entire energy of the Universe [11-14].

In connection with the conclusion as to the genetic unity of the electromagnetic and nuclear forces, note here that the intrinsic affinity between the electromagnetic and weak interactions, whose vehicles are vector bosons - photons and heavy bosons, respectively, is of different nature. These interactions prove to be a unified electroweak interaction only at energies on the order of $100 \mathrm{GeV}$, which is commensurable with the rest energy of intermediate vector bosons [45]. However, one can assume that it is the fluctuations of Casimir's field (8a), associated with the intrinsic dynamics of nuclei, that initiate the emergence of virtual vector bosons and effective realization of four-fermion interactions leading to weak nuclear processes at low energies. Thus, here is in fact introduced some generalized image of the electromagnetic component of the physical vacuum that involves a substantial proportion of the entire energy of the Universe and initiates manifestation of strong and maybe weak interactions by exerting effect on every nucleus as an open system. In what follows, it is demonstrated how the aggregate of the above-mentioned interactions is naturally supplemented with gravitation whose essence is also directly associated with the polarization of the QED vacuum in the neighborhood of material objects.

\section{Phenomenological Relations for the Fundamental and Interaction Constants}

Let us use the formal, "cosmological" representation of the Planck constant $\hbar$ that was introduced by Weinberg [50] who paid attention to the approximate equality

$$
\hbar \approx \frac{1}{2 \pi} G^{1 / 2} m_{\pi}^{3 / 2} R_{H}^{1 / 2}
$$

where $G$ is the gravitational constant, $R_{\mathrm{H}}=c / H$ is the Hubble radius (estimator of the radius of the Universe), $H$ is the Hubble constant (estimator of the age of the Universe, $t=$ $H^{-1}$ ), and $m_{\pi}$ is the mass of the $\pi$-meson. Such a numerological representation of the Planck constant proves heuristically useful, for it helps one to comprehend the cosmological essence of the law of universal gravitation. To this end, it will be expedient to express the Planck constant $\hbar$ in a different way:

$$
\hbar=\frac{1}{2 \pi} G^{1 / 2} m_{Q}^{3 / 2} R_{H}^{1 / 2}=\frac{G^{1 / 2} E_{Q}^{3 / 2}}{2 \pi c^{5 / 2} H^{1 / 2}}
$$

Introduced here is a new energy parameter $-E_{\mathrm{Q}}=m_{\mathrm{Q}} c^{2} \approx$ 209.5 MeV - whose value is set such that expression (11) is not an approximate, but exact equality involving the well-known relation between the de Broglie wavelength and the particle momentum. In that case, the parameter $E_{\mathrm{Q}}$ can be treated as the specific energy of alteration of the physical vacuum that corresponds to the elementary quantum of action. It should be noted that the value found for the parameter $E_{\mathrm{Q}}$ turns out to be correspondent to the energy scale of the quantity $E_{\mathrm{QCD}}$ considered in quantum chromodynamics. It is nuclear temperatures corresponding to the energy $E_{\mathrm{QCD}} \sim 200 \mathrm{MeV}$ that are critical to the phase transition whereby quarks inside the nucleus cease to be bound in nucleons, so that a quark-gluon plasma is formed. It is exactly this parameter that is considered as a characteristic quantity determining the masses of light quarks, protons, neutrons, and $\rho$-mesons [11, 12, 45, 51].

It also proves expedient to represent expression (11) in the form

$$
G=\frac{(2 \pi \hbar)^{2} H}{m_{Q}^{3} c}=\frac{8 \pi^{2} c H}{m_{Q}} a_{Q}^{2}=\frac{8 \pi^{2} c^{2} a_{Q}^{2}}{m_{Q} R_{H}}
$$

Here $a_{\mathrm{Q}}=2^{1 / 2} \hbar / m_{\mathrm{Q}} c \approx 1.3 \times 10^{-13} \mathrm{~cm}=1.3$ fermi is the "Bohr radius" $(9 \mathrm{a})$ associated with the mass $m_{\mathrm{Q}}$. The quantity $m_{\mathrm{Q}}=$ $E_{\mathrm{Q}} / c^{2} \approx 3.72 \times 10^{-25} \mathrm{~g}$ found above can be assigned the meaning of the elementary "gravitational" mass. In that case, it proves expedient to normalize the $i$ th particle masses $m_{i}$ appearing in the universal gravitation equation to $m_{\mathrm{Q}}$ and treat the quantity $\mu_{i}=m_{i} / m_{\mathrm{Q}}$ as the relative mass of the $i$ th particle. The quantity $q_{\mathrm{g}}^{2} \equiv G m_{\mathrm{Q}}{ }^{2}$ then can be treated as the squared elementary "gravitational" charge and, considering expressions (11a) and (9b), introduce the dimensionless gravitational interaction constant $\alpha_{\mathrm{g}}$ defined as

$$
\begin{aligned}
& \alpha_{g}=\frac{G m_{Q}^{2}}{\hbar c}=(2 \pi)^{2} \frac{\hbar H}{m_{Q} c^{2}}=2 \pi^{2} \frac{a_{Q}}{R_{H}} \alpha_{s}= \\
& =2 \pi^{2} \frac{\tau_{Q}}{H^{-1}} \alpha_{s}=\pi\left(\frac{E_{Q}}{E_{t o t}}\right)^{1 / 2} \alpha_{s}=\frac{q_{g}^{2}}{q_{s}^{2}} \alpha_{s} \approx 3.08 \times 10^{-40}
\end{aligned}
$$


In this expression, $\tau_{\mathrm{Q}}=\alpha_{\mathrm{Q}} / c \approx 4.34 \times 10^{-24} \mathrm{~s}$ stands for the characteristic "Bohr time" associated with the mass $m_{\mathrm{Q}}$ and the quantity $E_{\text {tot }}$ is introduced to denote the total energy content of the Universe at its specified age of $t=H^{-1}$ [15], defined as

$$
E_{t o t}=\frac{c^{5}}{2 G} \cdot \frac{1}{H} .
$$

Expression (12) reflects the quantum essence of the gravitational interaction $\left(\alpha_{g} \sim \hbar\right)$. According to the ideas being expounded, gravitation owes to the formation around each material particle of an attractive field for other particles as a consequence of the polarization of the physical vacuum in the vicinity of this particle. Such attractive fields, characterized by an infinite radius of action, make the potential barrier between particles approaching one another lower. For this reason, if the particles are free, there develops a component (drift) of their attractive force. The particles can merge, provided that this process is energetically advantageous. Hence it also follows that the propagation rate of gravitational interactions should be related not to the velocity of light in vacuum, $c$, but to the relaxation rate of the QED vacuum, which, as noted earlier in connection with the investigations [2,7], substantially exceeds $c$. As follows from expressions (12), the quantity $\alpha_{\mathrm{g}}$ is 38 orders of magnitude smaller than the fine structure constant $\alpha_{\mathrm{e}}$. The reason for such smallness is obvious: it is the smallness of the ratio between the characteristic size of the polarization region of the QED vacuum in the vicinity of the particle of "elementary gravitational mass" and the characteristic size of the Universe. The name "law of universal gravitation" given to the empirical relationship describing the gravitational interaction of two arbitrary masses is thereby "justified" at the conceptual level.

For comparison, let us present the quantity squared "elementary weak interaction charge" $q_{\mathrm{F}}{ }^{2} \equiv G_{\mathrm{F}} / a_{\mathrm{Q}}{ }^{2}$, where $G_{\mathrm{F}}$ $=1.436 \times 10^{-49} \mathrm{erg} \mathrm{cm}^{3} \approx 1.17 \times 10^{-5}(\hbar c)^{3} \mathrm{GeV}^{-2}$ is the Fermi four-fermion interaction constant $[45,52]$, and the corresponding dimensionless constant

$$
\alpha_{F}=\frac{q_{F}^{2}}{\hbar c}=\frac{1}{\sqrt{2}} \frac{a_{F}^{2}}{a_{Q}^{2}} \alpha_{s} \approx 2.810^{-5}
$$

where $a_{\mathrm{F}}=\left(G_{\mathrm{F}} / \hbar c\right)^{1 / 2} \approx 0.69 \times 10^{-16} \mathrm{~cm}$. We also present the following expression for the energy parameter $\eta$ of the scalar field in the standard electroweak interaction theory [52]:

$$
\eta=\frac{(\hbar c)^{3 / 2}}{2^{1 / 4} G_{F}^{1 / 2}}=\frac{\hbar c}{2^{1 / 4} a_{F}}=\frac{\hbar}{2^{1 / 4} \tau_{F}} \approx 246 \mathrm{GeV}
$$

where $\tau_{\mathrm{F}}=a_{\mathrm{F}} / c \approx 2.3 \times 10^{-27} \mathrm{~s}$. Note that the mass of the Higgs boson is expressed in terms of the parameter $\eta$ as $m_{\mathrm{H}}$ $=\lambda_{\mathrm{H}} \eta / c^{2}$, where $\lambda_{\mathrm{H}}$ is a dimensionless parameter (see Ref. [52]). It follows from the experiments conducted at the
Large Hadron Collider (LHC) $[32,33]$ that the mass of the Higgs boson is $m_{\mathrm{H}}=125.5 \mathrm{GeV} / c^{2}$, and so $\lambda_{\mathrm{H}} \approx 0.51$. Let us also write down the expressions for the quantities $\beta_{i \mathrm{~s}}-$ the ratios between the squared elementary charges $i$ of the interactions considered above $(i=\mathrm{e}, \mathrm{F}, \mathrm{g})$ and the squared elementary strong interaction charge $q_{\mathrm{s}}^{2}=\sqrt{2} \hbar c$ :

$$
\begin{aligned}
& \beta_{\mathrm{es}}=\frac{q_{\mathrm{e}}^{2}}{q_{\mathrm{s}}^{2}}=0.516 \times 10^{-2}, \beta_{\mathrm{Fs}}=\frac{q_{\mathrm{F}}^{2}}{q_{\mathrm{s}}^{2}}=0.74 \times 10^{-6}, \\
& \beta_{\mathrm{gs}}=\frac{q_{\mathrm{g}}^{2}}{q_{\mathrm{s}}^{2}}=2.05 \times 10^{-40} .
\end{aligned}
$$

\section{Conclusion}

According to the hypothesis as to the polarization of the QED vacuum in the vicinity of elementary particles, every particle of mass $m_{i}$ turns out to be related to the QED vacuum by the energy $E_{i}=m_{i} c^{2}$, with attractive gravitational fields being formed around every particle, which unite, thanks to the infinitude of their radius of action, all the material objects of our World into a unified system, thus minimizing the total energy of the Universe. It should be borne in mind that according to Weizsäcker's logic [8], the dynamics of the Universe inevitably involves a great many of various irreversible dissipative processes. The problems arising in that case with the compensation for the dissipated energy, as well as with the very fact of the presence of atomic nuclei as open systems sustaining their existence at the expense of the energy of the QED vacuum, prove to be resolvable in the open Universe model [15, 49] whose dynamics is governed by an energy-mass source constantly acting since the onset of the Big Bang.

The introduction of the notions of the polarization the QED vacuum in the neighborhood of elementary particles adds new dimensions to the understanding of the physical essence of a number of postulates of quantum mechanics and quantum electrodynamics. For example, when a microparticle in the form of a "vacuum polaron" gets localized in some concrete region of space (in a "box" of fixed size, as is the case with an electron localized at a proton to form a hydrogen atom), the polaron "coat" must fit into this volume, having distorted somehow to meet the "boundary conditions". It is precisely from such distortions that one can infer the genesis of the "activity" of the spatial coordinate associated in quantum mechanics with an operator $[15,21]$. No time discretization appears here; time can vary incessantly. It is for this reason that no time operator is objectively introduced in quantum mechanics. Another example is the introduction into the image of a virtual particle of the notions that it causes either no or but an incomplete polarization of the QED vacuum, so that its rest mass $m_{0}$ does not manifest itself in full (relation (9)), and therefore relation (2), valid for a free particle, does not hold. It can be supposed that the opinions stated in this work, along with the relations presented, can serve as a phenomenological benchmark for the future theory of the physical vacuum, into which the future theory of gravitation 
will enter as a natural fragment.

\section{References}

[1] L.D. Landau, and E.M. Lifshitz, The Classic Theory of Fields (Butterworth-Heinemann, Oxford, 4th Ed., 1975).

[2] Einstein A., Podolsky B., Rosen N. Can quantum-mechanical description of physical reality be considered complete? // Phys. Rev. 1935. V. 47. P. 777-780.

[3] Bell J.S. On the Einstein-Podolsky-Rosen paradox // Physics. 1964. V. 1. P. 195-200.

[4] B. B. Kadomtsev, Dynamics and information (Editorial office "Physics - Uspekhi", Moscow, 1997).

[5] Gröblacher S., et al An experimental test of non-local realism // Nature. 2007. V. 446. P. 871-875.

[6] Aspect A. To be or not to be local // Nature. 2007. V. 446. P. 866-867.

[7] Salart D., et al Testing the speed of "spooky action at a distance". Nature. 2008. V. 454. P. 861-864.

[8] Weizsäcker C.F. von. Time - Empirical Mathematics Quantum Theory // Time, Temporality, Now. Experiencing Time and Concept of Time in an Interdisciplinary Perspective / Editors: Harald Atmanspacher, Eva Ruhnau. - Berlin, Heidelberg, New York: Springer-Verlag, 1997. P.91-104.

[9] Silverman A. Resolution of a cosmological paradox using concepts from general relativity theory// Amer. J. Phys. 1986. V. 54. N 12. P. 1092-1096.

[10] Davis T.M., Lineweaver C.H., Webb J.K. Solutions to the tethered galaxy problem in an expanding universe and the observation of receding blueshifted object // Amer. J. Phys. 2003. V. 71. N 4. P. 358-364.

[11] Rubakov V.A. Hierarchies of fundamental constants (to items Nos 16, 17, and 27 from Ginzburg's list) // Physics - Uspekhi. Advances in Physical Sci. 2007. V. 50. N 4. P. 390-396.

[12] Sahni V. Dark matter and dark energy // Lect. Notes Phys. 2004. V. 653. P. 141-180; arxiv.org/abs/astro-ph/0403324v3.

[13] Chernin A.D. Dark energy and universal antigravitation // Physics - Uspekhi. Advances in Physical Sci. 2008. V. 51. N 3. P. 253-282.

[14] Padmanabhan T. Darker side of the Universe. 29 International Cosmic Ray Conf. Pune. 2005. V. 10. P. 47-62.

[15] Timashev S.F. Physical vaccum as a system manifesting itself on various scales - from nuclear physics to cosmology: http://arxiv.org/abs/1107.1799v7.

[16] G.W. Erickson. Improved Lamb-shift calculation for all values of Z // Phys. Rev. Lett. 1971. V. 27. P. 780-783.

[17] Klimchitskaya G.L., Mohideen U., Mostepanenko V.M. The Casimir force between real materials: experiment and theory // Rev Mod. Phys. 2009. V. 81. P. 1827-1885.

[18] Mostepanenko V.M., Trunov N.N. The Casimir effect and its applications // Physics - Uspekhi. Advances in Physical Sciences. 1988. V. 31. N 3. P. 965-987.
[19] C. Beck, M.C. Mackey. Zeropoint fluctuations and dark energy in Josephson junctions // Fluctuation and Noise Letters. 2007. V. 7. No. 2. P. C27-C35.

[20] Burlankov D.E. Time, space, gravity. Moscow-Izhevsk: NIC "Regular and Chaotic Dynamics". 2006. 420 p. (in Russian).

[21] Timashev S.F. On the microscopic origin of the Second Law // Quantum Limits to the Second Law. First International Conference on Quantum Limits to the Second Law. AIP Conference Proceedings: V. 643 / Editor Daniel P. Sheehan. Melville, New York: Amer. Inst. Phys., 2002. - P. 367-372.

[22] Okun' L.B. The Einstein formula: $E_{0}=m c^{2}$. Is not the Lord laughing? // Physics - Uspekhi. Advances in Physical Sciences. 2008. V. 51. N 5. P. 513-527.

[23] Feynman R.P., Leighton R.B., Sands M. The Feynman Lectures on Physics. Vol. 1: Mechanics. Heat. Radiation. Chap. 15, § 9. Addison Wesley Reading Mass. 1966.

[24] Thomson J.J. On relation between matter and ether in accordance with the novel researches in the electricity. The speech given on the $4^{\text {th }}$ of November 1907 at Manchester University. In: Ether and matter. Moscow: URSS. 2004. P. 71-92. (in Russian).

[25] Ovchinnikov A.A., Timashev S.F., and Belyy A. A. Kinetics of Diffusion Controlled Chemical Processes (Nova Science Publishers, New York, 1989), 239 pp.

[26] Timashev S.F., Polyakov Yu.S., Misurkin P.I., Lakeev S.G. Anomalous diffusion as a stochastic component in the dynamics of complex processes // Physical Rev. E. 2010. V. 81. No 4. P. 041128-1-17; http://arxiv.org/abs/1004.0235

[27] Brooks D.W.C., Botter T, Schreppler S. Non-clqssic light generated by quantum-noise-driven cavity optomechanics // Nature. 2012. V.488. P. 476-480.

[28] Dalvit D.A.R., Neto P.A.M., Mazzitelli F.D. Fluctuations, dissipation and the dynamical Casimir effect. http://arxiv.org/abs/1006.4790v2.

[29] Wilson C.M., Johansson G., Pourkabirian A., Johansson J.R., Duty T., Nori F., Delsing P. Observation of the Dynamical Casimir Effect in a Superconducting Circuit. http://arxiv.org/pdf/1105.4714v1

[30] Pauli W. Theory of relativity. Translated by G. Field. New York: Pergamon Press Inc., 1959. 241 pp

[31] Higgs P.W. Broken symmetries, massless particlees and gauge fields // Phys. Lett. 1964. V. 12. P. 132-133.

[32] The CMS Collaboration. Observation of a new boson at a mass of $125 \mathrm{GeV}$ with the CMS experiment at the LHC. http:// 1207.7235v2 [hep-ex].

[33] The ATLAS Collaboration. Observation of a New Particle in the Search for the Standard Model Higgs Boson with the ATLAS Detector at the LHC. http:// 1207.7214v2 [hep-ex]

[34] Ginzburg V.L. Radiation by uniformly moving sources (Vavilov-Cherenkov effect, transition radiation, and other phenomena) // Physics - Uspekhi. Advances in Physical Sciences. 1996. V. 39. N 10. P. 973-982.

[35] Bolotovskii B.M. Vavilov-Cherenkov radiation: its discovery and application // Physics - Uspekhi. Advances in Physical Sciences. 2009. V. 52. N 11. P. 1099-1110. 
[36] Bailey J., Borer K., Combley F., et al. Measurements of relativistic time dilatation for positive and negative muons in circular orbit // Nature. 1977. V. 268. P. 301-305.

[37] Boyer T.H. Quantum electromagnetic zero-point energy of a conducting spherical shell and the Casimir model for a charged particle // Phys, Rev. 1968. V. 174. P. 1764-1776.

[38] Feynman R.P., Leighton R.B., Sands M. The Feynman Lectures on Physics. Vol. 2: Electromagnetism and Matter. Chap. 28, § 4. Addison Wesley. Reading Mass. 1966.

[39] Milton K.A., DeRaad L.L., Jr, Schwinger J. Casimir self-stress on a perfectly conducting spherical shell // Annals of Physics. 1978. V. 115. P. 388-403.

[40] Ohanian H.C. What is spin? // American Journal of Physics. 1986. V. 54. N 6. P. 500-505.

[41] Migdal A.B. Quadrupole and dipole $\gamma$-radiation of nuclei // JETP. 1945. V. 15. P. 81-88 (in Russian).

[42] Ferentz M., Gell-Mann M., Pines D. The giant nuclear dipole resonance // Phys. Rev. 1953. V. 92. P. 836-837.

[43] Ishkhanov B.S., Yudin N.P., Eramzhyan R.A. Giant Resonances in Atomic Nuclei // Physics of Elementary Particles and Atomic Nuclei. 2000. V. 31. N. 2. P. 313-349.
[44] Landau L.D., Lifshitz E.M. Quantum Mechanics: Non-Relativistic Theory. Oxford: Pergamon Press, 1977.

[45] Bettini A. Introduction to elementary particle Physics. Cambridge: Cambridge University Press. 2008, 431 p.

[46] Dubovikov M.S., Simonov Yu.A. Decay of resonant states and determination of their quantum numbers // Physics Uspekhi. Advances in Phys. Sci. 1971. V. 13. N 4. P. 522-543.

[47] Lanczos C. The Variational Principles of Mechanics. Toronto: Univ. Toronto Press, 1964.

[48] Dirac P.A.M. Principles of Quantum Mechanics. Oxford University Press. 1982. 4th Edition. 314 p.

[49] Timashev S.F. Planckian energy-mass source and the Universe dynamics: phenomenology // Paper 2.

[50] Weinberg S. Cosmology. Oxford: Univ. Press. 2008, 593 p.

[51] Ioffe B.L. The origin of mass and experiments on high-energy particle accelerators // Physics - Uspekhi. Advances in Phys. Sci. 2006. V. 49. N 10. P. 1077-1078.

[52] Okun' L.B. Physics of elementary particles. Moscow: Nauka. 1984, 224 p. (in Russian) 